\title{
Formulation, evaluation and anti-microbial potential of topical Licorice root extract gel
}

\author{
Amina Mudhafar Al-Nima1 ${ }^{*}$, Zahraa Sedeeq Qasim², Myasar Al-Kotaji ${ }^{1}$ \\ ${ }^{1}$ Department of Pharmaceutics, ${ }^{2}$ Department of Clinical Laboratory Sciences, College of \\ Pharmacy, University of Mosul, Mosul, Iraq \\ *Address for correspondence E-mail: \\ amnah.mudhafar@uomosul.edu.iq \\ Received Accepted \\ 5.11.2020 5.12.2020
}

\section{ABSTRACT}

Objectives: This work aims at formulating licorice as topical gel preparation and at exploring its antimicrobial efficacy against different types of microorganisms including two types of bacteria Staphylococcus aureus (S.aureus), Pseudomonas aeruginosa (P.aerueginosa) and one type of yeast, Candida albicans (C.albicans).

Materials and Method: Different gel formulas were prepared using two different types of gelling agent Carbopol 934 and HPMC (methocel E5) with different extract concentrations. All the prepared formulas were physically and chemically characterized, and the selected formula showed good appearance and consistency, spreadability, accepted $\mathrm{pH}$ and physiologically compatible to the skin. The selected formula was further characterized by studying the invitro antimicrobial activity.

Result and Discussion : Results showed that licorice gel can be formulated successfully using Carbopol 934 as gelling agent and the selected formula exhibited good antimicrobial activity against (S.aureus) and (C.albicans), but it showed no antimicrobial activity against (P.aerueginosa). Consequently, the prepared topical gel of licorice could be considered as an efficient alternative to the common topical antimicrobial agents. Furthermore, the results pointed out that the prepared licorice gel has good stability at room temperature with no significant difference $(\mathrm{p} \leq 0.05)$ after storing for four months.

Conclusion: In this work a suitable candidate of new herbal antimicrobial topical gel formulation was proposed to enter into the market. 
Key words: licorice, medicinal plant, antimicrobial study, extract, gel formula.

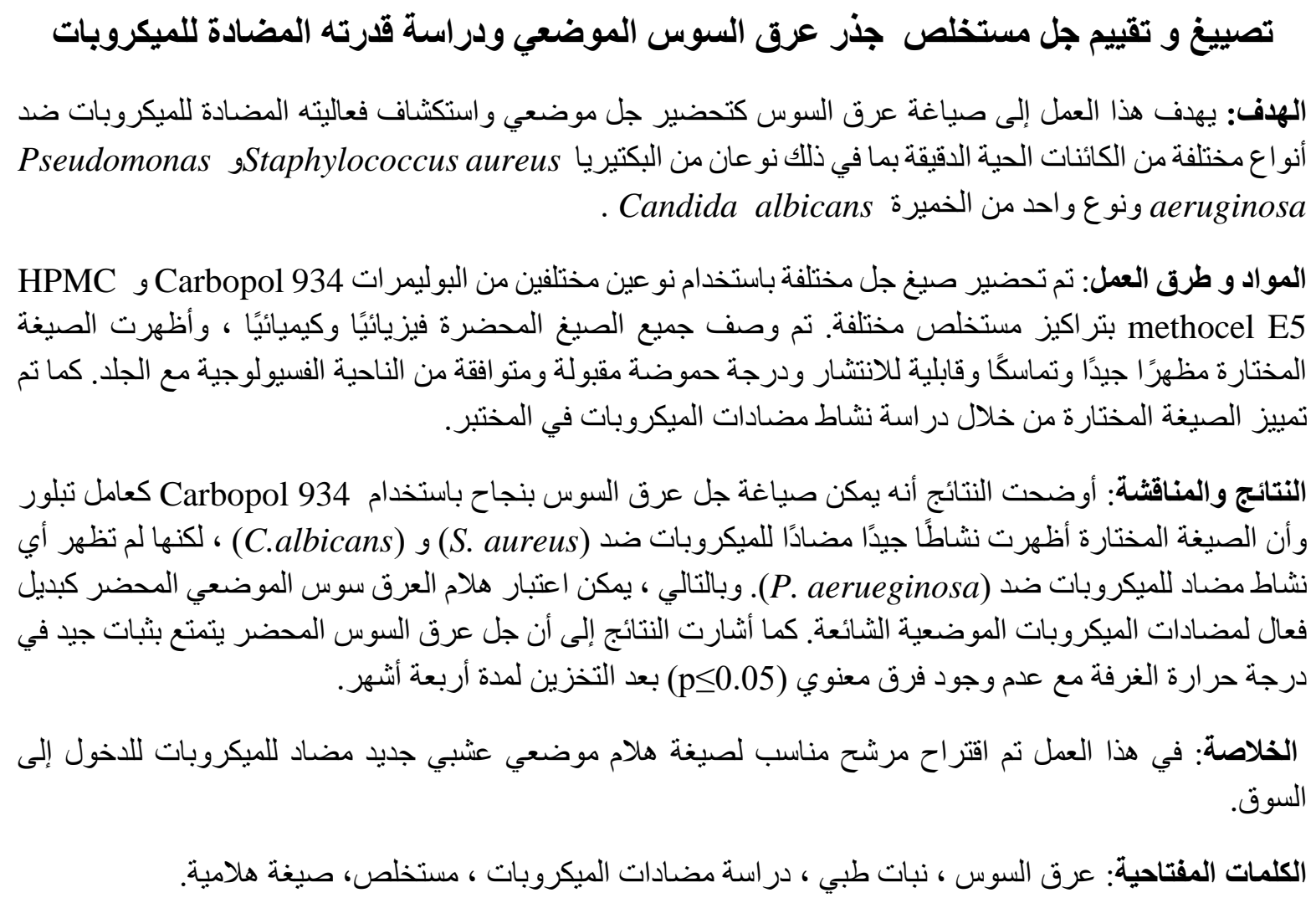

\section{INTRODUCTION}

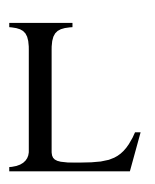
icorice (Glyceriza glabra) is considered as one of herbal medicine that is traditionally used for many years and for different applications. Licorice is approved by US Food and Drug Administration (FDA) as food supplement used in many products. ${ }^{1}$

There are widespread medicinal uses of licorice. It is used for management of peptic ulcers, asthma, malaria, pharyngitis, abdominal pain, insomnia and infections. There are numerous numbers of unique classes of molecular entities have been established in licorice root extract. Among these molecules are 20 triterpenoids and nearly 300 flavonoids. $^{2} \quad$ Triterpenoids are natural glycosides having important structure and bioactive variety. They are becoming significantly used in the treatment of cancer due to their safety and effectiveness. ${ }^{3,4}$ The effectiveness of licorice can be due to a number of mechanisms e.g. glycyrrhizin and glycyrrhizic acid which have been shown to suppress 
numerous RNA and DNA viruses growth, including hepatitis A9 and C, herpes zoster, HIV, Herpes simplex and CMV. Hepatic metabolism of aldosterone is suppressed by glycyrrhizin and its metabolites. There is similarity in structure of glycyrrhetic acid to that of hormones secreted by the adrenal cortex resulting in activity similar to the mineralocorticoid and glucocorticoid activity. Licorice constituents also show steroid like anti-inflammatory activity, which resembles the action of hydrocortisone. ${ }^{3,4}$

Glycyrrhizic acid inactivates cyclooxygenase and inhibits prostaglandin production (specifically prostaglandin E2). In addition, glycyrrhizic acid has the ability to suppress, indirectly, the platelet aggregation and all factors in the process of inflammatory, as demonstrated by in vitro study. The major active molecule in licorice is glycyrrhizic acid; in addition, it contains other important contents like flavonoids, isoflavonoids, coumarins, chalcones, triterpenoids, starch, sterols, sucrose, glucose, lignins, amines, amino acids, gums and volatile oils. ${ }^{5,6}$
Licorice have several other biological activities, which include antioxidative and antiprotozoal activity. In addition, licorice have been shown to be potential sources for new anti-microbial agents. ${ }^{7,8}$

The resistance to anti-microbial agents had increased worldwide, and this led to an urgency to develop recent anti-microbial compounds from different sources, such as medicinal plants. The survey for new medicaments with a greater antimicrobial activity and less toxicity had paved the way to introduce the herbal anti-microbials. Herbal antimicrobial could aid in controlling the resistant infections and aids in reducing the cost of drug regimens. ${ }^{9,10}$

Topical gel has gained wide acceptance worldwide due to its ease of application, non-greasy and easy to remove. Although several works were conducted on using licorice gel for treatment of dermatitis or aphthous ulceration ${ }^{11}$, there is no marketed topical gel of licorice in the Iraqi market.

This work has several aims, which include preparation of licorice gel, studying the physicochemical properties of this gel and studying 
the potential anti-microbial effects against different microorganisms that are potential cause of topical infection including two types of bacteria Gram positive (S.aureus) and Gram negative (P.aeruginosa), and one type of yeast (C.albicans).

\section{MATERIALS AND CHEMICALS}

The dried coarse powder of licorice root was purchased from local market in Mosul City, Iraq. Ethanol was procured from Tedia Company, USA. Carbopol 934 (CAR-934) was purchased from (HIMEDIA, India), Hydroxy propyl methyl cellulose (HPMC) methocel E5 was obtained from Pioneer Company for pharmaceutical industries, Sulaymaniyah, Iraq. Propylene glycol was purchased from (THOMAS BAKER Co., India). Methyl paraben and propyl paraben were purchased from (Direvo, Germany), triethanolamine was obtained from (Tedia, USA). All other chemicals were of analytical grade.

\section{Preparation of Licorice Gel using CAR-934}

Several gel formulas were prepared using different

\section{METHOD}

\section{Extraction of Licorice root}

Licorice root was pulverized into fine powder for two minutes using a mechanical grinder (RoyalJapan). The dried powder (100g) was soaked in one liter of $70 \%$ ethanol for two days for softening which enhances the extraction process. To accelerate the extraction process, the licorice powder-in-alcohol mixture was blended using an electric blender (Royal-Japan) for 10 minutes at room temperature, then the resulting extract was filtered through folded gauze and filtered again using Whatman filter papers. The filtrate was poured in glass petri dishes for evaporation in air to give a residue which will then be scratched, weighed and kept in an airtight closed container in a refrigerator. ${ }^{12,13}$

concentrations of CAR-934 as gelling polymer. These formulas are presented in Table 1, where code CAR is given for formulas prepared with CAR-934. The CAR-934 dispersion was 
prepared by adding $15 \mathrm{ml}$ distilled water to the polymer with slight hand-mixing and then stirred by using a magnetic stirrer (Fisher Scientific, Korea) at $350 \mathrm{rpm}$ with heating at about $50-55^{\circ} \mathrm{C}$ for $2-3$ hours. After cooling, the dispersion was covered with a piece of aluminum foil and left overnight to let expelling of air bubbles. The preservatives (methyl paraben and propyl paraben) were dissolved within propylene glycol using a different beaker and stirred using a magnetic stirrer (Fisher scientific, $400 \mathrm{rpm}, 25-30^{\circ} \mathrm{C}, 2-3$ hours). Then the preservative solution was mixed with polymer dispersion using a magnetic stirrer (400 rpm, 2 hours) to get a clear dispersion. The licorice extract powder was hand-mixed with little amount of distilled water for nearly 10 minutes until forming a one phase solution (extract solution) which was left in a refrigerator. In the next day, the extract solution was added to the mixture of both the preservative solution and the polymer dispersion and all were stirred together at $(350 \mathrm{rpm}, 2-3$ hours). As a final step of gel preparation, few drops of triethanolamine were added and the gel formula was left in a refrigerator. ${ }^{13,14,15}$

Table 1. Composition of licorice gel, code CAR is presenting formulas prepared with CAR-934. Formula (CAR-3) is the selected formula. 


\begin{tabular}{|l|c|c|c|c|}
\hline & CAR-1 & CAR-2 & CAR-3 & CAR-4 \\
\hline Licorice extract & $5 \%$ & $5 \%$ & $5 \%$ & $10 \%$ \\
\hline CAR-934 & $0.8 \%$ & $1 \%$ & $1.2 \%$ & $1.5 \%$ \\
\hline Propylene Glycol & $10 \%$ & $10 \%$ & $10 \%$ & $10 \%$ \\
\hline Methyl paraben & $0.02 \%$ & $0.02 \%$ & $0.02 \%$ & $0.02 \%$ \\
\hline Propyl paraben & $0.005 \%$ & $0.005 \%$ & $0.005 \%$ & $0.005 \%$ \\
\hline Triethanolamine & sq. & sq. & sq. & sq. \\
\hline
\end{tabular}

\section{Preparation of Licorice Gel using}

\section{HPMC}

Other formulas were prepared using different concentration of HPMC (methocel E5) as a gelling polymer and they are presented in Table 2 where Code $\mathrm{H}$ is given for formulas prepared with this polymer. HPMC dispersions were prepared by mixing one third of the final quantity of distilled water with the polymer in one beaker (350 rpm, $\quad 30^{\circ} \mathrm{C}, \quad 2-3$ hours). The preservatives beaker containing (methyl paraben and propyl paraben), these two preservatives were weighed, dissolved in propylene glycol and stirred at (400rpm, $25-30^{\circ} \mathrm{C}, 2$ hours). The extract solution was prepared in a different beaker by mixing the prepared dry powder licorice extract with little amount of water as mentioned above. In the next day the contents of the three beakers were added to each other forming the final licorice gel formula which was covered with a piece of aluminum foil and left in a refrigerator. ${ }^{15,16,17}$ 
Table 2. Composition of licorice gel, code $H$ is presenting formulas prepared with HPMC. Formula (H-4) is the selected formula.

\begin{tabular}{|l|c|c|c|c|c|}
\hline & H-1 & H-2 & H-3 & H-4 & HCAR \\
\hline Licorice extract & $5 \%$ & $5 \%$ & $5 \%$ & $5 \%$ & $5 \%$ \\
\hline CAR-934 & -- & -- & -- & -- & $1 \%$ \\
\hline HPMC methocel E5 & $2 \%$ & $5 \%$ & $10 \%$ & $15 \%$ & $2 \%$ \\
\hline Propylene Glycol & $10 \%$ & $10 \%$ & $10 \%$ & $10 \%$ & $10 \%$ \\
\hline Methyl paraben & $0.02 \%$ & $0.02 \%$ & $0.02 \%$ & $0.02 \%$ & $0.02 \%$ \\
\hline Propyl paraben & $0.005 \%$ & $0.005 \%$ & $0.005 \%$ & $0.005 \%$ & $0.005 \%$ \\
\hline Triethanolamine & -- & -- & -- & - & sq. \\
\hline
\end{tabular}

\section{Preparation of Licorice Gel using} a combination of CAR-934 and HPMC

HCAR formula was prepared using both HPMC polymer and CAR-934 polymer. Dispersions of each polymer in water were prepared separately and left overnight to exclude air bubbles. Then, these two dispersions with the preservative

EVALUATION OF LICORICE GEL

\section{Appearance and Homogeneity}

The prepared formulas were tested for appearance and homogeneity by a solution and licorice extract solution were mixed together with continuous stirring at $(350 \mathrm{rpm}, 40-$ $50^{\circ} \mathrm{C}, \quad 3-4$ hours), then triethanolamine was added in a sufficient quantity until forming a uniform gel. ${ }^{16,17}$ All above stirring procedure was accomplished with the use of magnetic stirrer (Fisher Scientific, Korea).

visual inspection after the gel had been filled in the container. Tests have been made for their color, appearance as gel and presence of aggregates. ${ }^{18,19}$

\section{Determination of $\mathrm{pH}$}


A $0.5 \mathrm{~g}$ gel was accurately weighed and dispersed in $50 \mathrm{ml}$ distilled water. The $\mathrm{pH}$ of the dispersion was measured after two hours using digital $\mathrm{pH}$ meter, which was calibrated using standard buffer solution at 4.0, 7.0 and 9.0. The measurements of $\mathrm{pH}$ were taken in triplicate and average values were calculated. ${ }^{17,20}$

\section{Skin irritation test}

This test was performed for all the prepared formulas to prove the compatibility of the gel with the skin. It was applied on human volunteers to check out if there is any irritancy problem which could make the gel inconvenient for use. Five human volunteers were chosen to check skin irritancy test. A $1 \mathrm{~g}$ of the tested gel was applied topically to the hand over a nearly 2 square inches. In this test, the five volunteers agreed to participate by signing an informed consent form. Observations for any redness, lesions, irritation, edema, and any sign of irritancy to the skin were performed at constant intervals for about $24 \mathrm{~h}$ and recorded. ${ }^{21,22}$

\section{Spreadability Test}

One of the criteria for a topical formula, especially gel formulas, is that it should have good spreadability. Spreadability is the term expressed to denote the extent of surface area to which formula readily spreads on application to skin or affected part. To determine the spreadability of a formula, simple apparatus was used; a home-made apparatus consists of two glass slides of the same dimensions and the spreadability was calculated on the basis of "slip" and "drag" characteristics of the gels. An excess of gel (around $2 \mathrm{~g}$ ) was placed on the surface of the ground slide and allowed to move, the time was recorded and the spreadability was determined as published elsewhere. ${ }^{15} \mathrm{~A}$ shorter time interval indicates preferablespreadability. Spreadability (S) was calculated through the following equation:

$$
\mathrm{S}=\mathrm{M} . \mathrm{L} / \mathrm{T}
$$

Where $S=$ spreadability, $M=$ mass tied to upper slide $(\mathrm{g}), \mathrm{L}=$ length of glass slide $(\mathrm{cm})$ and $\mathrm{T}=$ time taken (sec) by the slide to move the distance. ${ }^{17,23,24}$

\section{Viscosity Determination}

Viscosity is an important feature of the gel formula because it denotes the resistance of gel to flow when applied on the skin surface. It was determined for the best gel formulas by using a 
viscometer (NDJ-5S, China) supplied with spindle 3 and $4 .^{23,25}$

\section{Selection of the Best Gel Formula}

One of the main or basic ingredients of the formula is the gelling agent (polymer). In order to select the type and concentration of polymer, gel formulas were prepared with two different polymers, CAR-934 and HPMC. Different concentrations of these polymers were tried alone and in combination and different concentrations of the extract were also used as shown in table 1 and 2. The selection of the final gel formula depends on the following criteria: good appearance, consistency, spreadability, suitable $\mathrm{pH}$ and lack of irritancy to skin. The selected formula has been further evaluated for potential anti-microbial effect.

\section{Stability Study for the Selected Licorice Gel Formula}

The selected licorice gel formula, which had been kept in a well-closed container, was tested for stability which was performed at $25 \pm 2^{\circ} \mathrm{C}$ for 4 months. Samples were observed at an ascertained time interval of 1,2,3 and 4 months. At the end of 4 months, the elected formula was evaluated for its physical properties including mainly: appearance, color, presence of clogs or any aggregates, the consistency and spreadability. The elected formula was also evaluated for other parameters like variation in $\mathrm{pH} .{ }^{17,26,27}$

\section{In Vitro Anti-microbial Activity}

The microorganisms used in this study (Staphylococcus aureus, Pseudomonas aeruginosa and Candida albicans) were obtained from microbiology laboratory of clinical laboratory sciences department, College of pharmacy, University of Mosul. The antimicrobial activity of licorice gel was evaluated using disc agar diffusion method. The obtained bacteria were subcultured on nutrient agar, while the yeast was subcultured on Sabouraud dextrose agar and then plates were incubated aerobically for 24 hours at $37^{\circ} \mathrm{C}$ for bacteria and for 48-72 hours at $37^{\circ} \mathrm{C}$ for yeast.

\section{Anti-microbial Activity}

\section{Susceptibility Test}

Susceptibility test was applied on two types of bacteria; S. aureus as a Gram positive bacteria and p.aerueginosa as a Gram negative bacteria and one type of yeast (C. albicans). The susceptibility test for bacteria was processed by mixing 4-5 of pure 
culture colonies of bacteria into 3-5 $\mathrm{ml}$ Muller-Hinton broth, then bacterial suspension was achieved 0.5 MacFarland standard visually. A cotton swab was soaked into bacterial inoculum suspension and then it was streaked over a whole dried surface (90 mm Muller- Hinton agar) plate. On the other hand, the susceptibility test for yeast was processed by mixing portion of pure culture colony of $C$. albicans into Sabouraud dextrose broth, then yeast suspension was achieved 0.5 MacFarland standard visually. A cotton swab was soaked into yeast inoculum suspension, and then it was streaked over a whole dried surface $(90 \mathrm{~mm}$ Sabouraud dextrose agar) plate.

\section{Preparation of Discs}

A stock concentration was prepared from $5 \%$ licorice gel by dissolving $200 \mathrm{mg}$ in $1 \mathrm{ml}$ of distilled water, discs were prepared from sterile filter paper Whatman No.1 (6mm radius) thatsaturated with different concentrations of $5 \%$ licorice gel prepared from stock concentration $(5,10,15,20,25,30 \mathrm{mg} / \mathrm{ml})$ and kept overnight for saturation. ${ }^{28}$ The discs were applied on the inoculated Muller-Hinton and Sabouraud dextrose agar by sterile forceps, then the plates were inoculated for 24hours for bacteria and 24-48 hours for C. albicans at $37^{\circ} \mathrm{C}$. The diameter of inhibition zone was measured for the assessment of anti-microbial activity; the diameter was measured in unit of millimeter. Chloramphenicol $10 \mu \mathrm{g}$ were tested on both types of bacteria while voriconazole $1 \mu \mathrm{g}$ was tested for Candida albicans as control antibiotic discs. The susceptibility test was carried out in triplicate to ensure the reliability and the mean of the susceptibility test were calculated. $^{29,30}$

\section{Statistical Analysis}

The results were taken as mean \pm standard deviation and statistically analyzed using t-test. A value of $p<$ 0.05 was considered significant.

\section{RESULTS AND DISCUSSION}

\section{Evaluation of Licorice Gel}

The prepared gel has been prepared from licorice root extract. The extraction was carried out by simple method (hydroalcoholic extraction) and it was easy except the process of scratching from the petri dishes; however, the yield of extracting $100 \mathrm{~g}$ in one liter $70 \%$ ethanol is 10 grams 
only. It is worthy to mention that the problem of low yield value necessitates more efficient method of extraction or trying different solvent to overcome the problem.

\section{Selection of Gel with}

\section{Considerations of Visual}

\section{Appearance}

Different formulas were prepared, taking in consideration the main ingredient of the formula, the type and concentration of gelling agent. The concentration of the latter is of a distinctive value as low gelling agent concentration will lead to simple solution or lotion characterizing by very low consistency, while high gelling agent concentration may result in formation of high viscosity gels leading to low uniformity of the extract distribution and a difficulty in handling of gel. Different gelling agents were tested to select the best gelling agent. Gels containing combination of CAR-934 and HPMC methocel E5 showed white color as shown in figure (1a), which might be due to possible interaction between the two polymers with the extract, so they were excluded from further experiments.

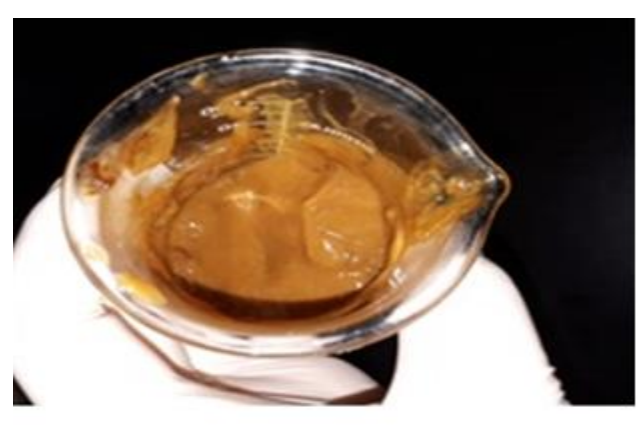

Figure (1a)

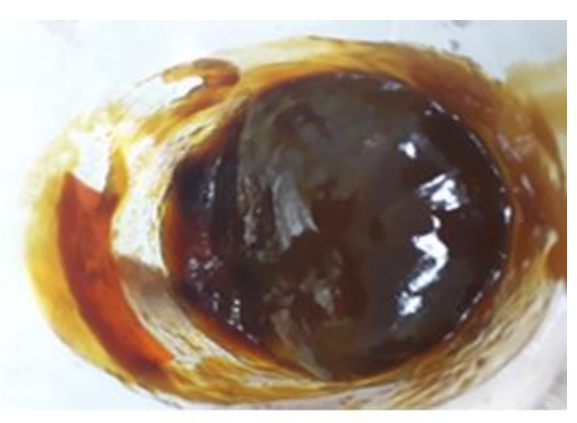

Figure (1b)

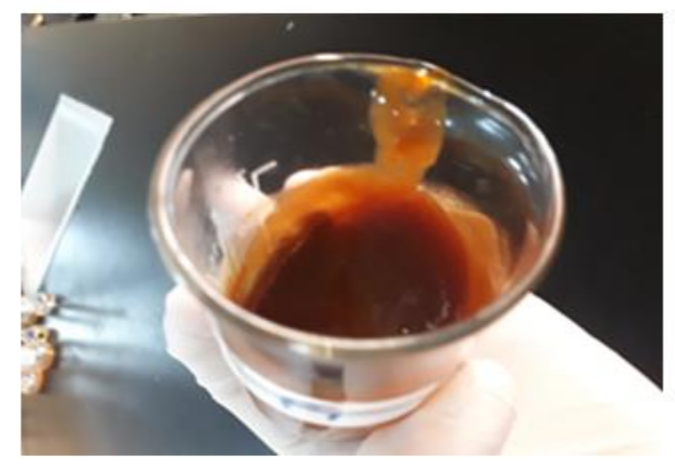

Figure (1c) 
Figure(1a) Licorice gel containing combination of CAR-934 and HPMC methocel E5 (HCAR formula)

Figure(1b) Licorice gel containing 1.2\% of CAR-934 (CAR-3 formula)

Figure(1c) Licorice gel containing 15\% HPMC methocel E5 (H-4 formula)

In addition to the effect of type of gelling agent on the prepared formulas, the effect of different concentrations of licorice extract were also investigated. Two concentrations were tried $(5 \%$ and $10 \%)$ as shown in tables (1 and 2), and it was observed that using high concentration (10\% extract) led to difficulty in the formation of gel, especially when using CAR-934 as a gelling agent. The possible reasons for that might be the presence of glycyrrhizic acid, the main component of licorice extract as stated by Trupti W. et al. ${ }^{31}$ The presence of glycyrrhizic acid may counteract the basicity which was desirable for gel formation when CAR-934 was used. High extract concentration $(10 \%)$ was not tried with HPMC methocel E5 polymer.

Concerning the effect of concentration of gelling agent, licorice gels containing less than 1.0 $\%$ of CAR-934 (CAR-1 formula) formed a very thin gel that liquefied within $6 \mathrm{~h}$ of preparation, so it was excluded from further work. When
1.2\% CAR-934 (CAR-3 formula) was used as gelling agent, a uniform and smooth gel with no problem of liquefaction was obtained (see Figure 1b). Gel containing $1.5 \%$ of CAR934 (CAR-4 formula) formed very thick and sticky formula that could not be properly spread out and could not even move from its place in the container. Thus CAR-3 formula was selected as the successful formula, which was further evaluated for its $\mathrm{pH}$, viscosity, spreadability and stability.

Another set of formulas prepared With HPMC (methocel E5). The gels formed are low in consistency and have low viscosity especially with low HPMC concentrations like formulas $\mathrm{H}-1, \mathrm{H}-2$ and $\mathrm{H}-3$, which were prepared using $2 \%, 5 \%$ and $10 \%$ of HPMC, respectively. Accordingly, formulas $\mathrm{H}-1, \mathrm{H}-2$ and $\mathrm{H}-3$ were excluded from further characterizations.

Higher concentration was used $(15 \%$ of HPMC) and the gel formed (H-4 formula) was smooth, uniform, accepted as a gel and it was selected 
as the best formula containing HPMC, further evaluations were conducted on this formula, see Figure (1c).

\section{Determination of $\mathbf{p H}$}

The $\mathrm{pH}$ of the formulas (CAR-3 and $\mathrm{H}-4$ ) was determined for assuring that the formula can be used with no risk of skin irritancy. The $\mathrm{pH}$ was determined to be $5.5 \pm 0.5$ which was close to the required $\mathrm{pH}$ for topical preparation, thus the formulas can be used with no risk of skin irritancy. This also indicated that the selected ingredients of the formula did not change the $p \mathrm{H}$ of the formula.

\section{Skin Irritancy Test}

After doing this test and observations for any undesirable effects at constant intervals for about $24 \mathrm{~h}$, results represented that there were no redness, no edema and no irritation or other unwanted effects after application.

\section{Spreadability Test}

The spreadability of formulas was found to be decreased with increasing the gelling agent concentration. The spreadability value for the best gel in the present study was measured to be in the range of $0.45-0.89 \mathrm{~g} . \mathrm{cm} / \mathrm{sec}$, which indicates that the gel is easily spreadable by small amount of shear. The results indicated that the formulas (CAR-3 and H-4) can be applied easily without being running off. This assures that the formulas maintain an acceptable wet contact time when applied to the application site of skin.

\section{Viscosity Determination}

Regarding viscosity determinations of the two formulas (CAR-3 and H-4), two types of spindle were used. For CAR-3, spindle 3 was used, while spindle 4 was used for H-4. Results showed that Formula $\mathrm{H}-4$ exhibited higher viscosity than formula CAR-3 as demonstrated in table 3 .

Table3. Viscosity determination of CAR-3 and H-4 formulas

\begin{tabular}{|c|c|c|c|}
\hline \multicolumn{2}{|c|}{ Formula CAR-3 } & \multicolumn{2}{c|}{ Formula H-4 } \\
\hline $\begin{array}{c}\text { Shear force } \\
\text { (1pm) }\end{array}$ & viscosity (mPa.s) & $\begin{array}{c}\text { Shear force } \\
\text { (1pm) }\end{array}$ & viscosity (mPa.s) \\
\hline 6 & 14404 & 6 & 52067 \\
\hline 12 & 8005.2 & 12 & 43749 \\
\hline
\end{tabular}


Stability Study for the Selected

\section{Licorice Gel Formulas}

When the selected formulas (CAR-3 and $\mathrm{H}-4$ ) were kept firmly closed and stored up at room temperature for 4 months, there was no significant difference $\quad(p \leq 0.05) \quad$ in visual appearance, color, uniformity, consistency, spreadability and $\mathrm{pH}$ was observed. This indicated that these formulas are stable when stored at room temperature.

\section{Anti-microbial Activity}

Formula CAR-3, was selected to investigate the antimicrobial efficacy

Table 4. Susceptibility test for $5 \%$ licorice gel against different types of microorganisms.

\begin{tabular}{|c|c|c|c|c|c|c|c|c|}
\hline \multirow{3}{*}{ Microorganisms } & \multicolumn{6}{|c|}{$5 \%$ Licorice gel concentrations } & \multicolumn{2}{|c|}{ Control } \\
\hline & 5 & 10 & 15 & 20 & 25 & 30 & *Chloram. & **Vorico. \\
\hline & \multicolumn{8}{|c|}{ Zone of inhibition (mm) } \\
\hline S. aureus & 0 & 12 & 15 & 20 & 25 & 25 & 18 & -- \\
\hline$P$. aenuginosa & 0 & 0 & 0 & 0 & 0 & 0 & 25 & -- \\
\hline C. albicans & 12 & 13 & 13 & 25 & 25 & 30 & - & 25 \\
\hline
\end{tabular}

*Chloram. $=$ chloramphenicol, $* *$ Vorico $=$ voriconazole 


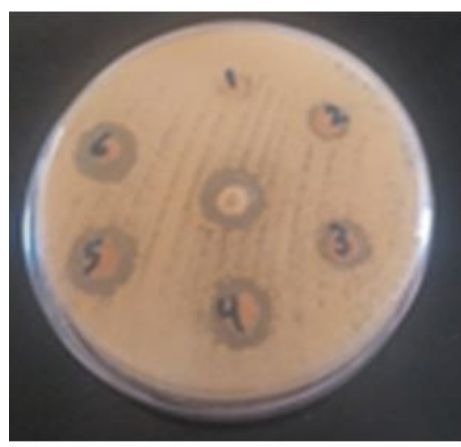

S. aureus

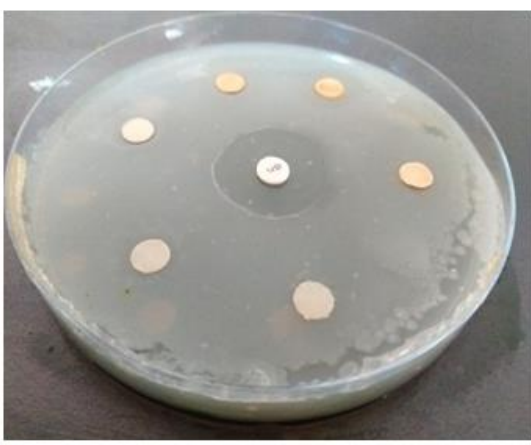

P. aureginosa

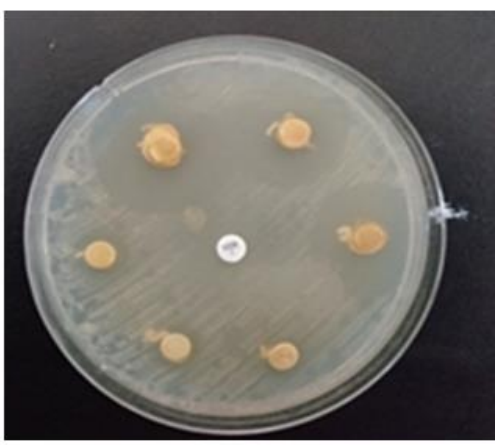

C. albicans

Figure (2): Susceptability test showed zone of inhibition of $5 \%$ licorice gel against S.aureus. S.aeruginosa, and C.albicans.

According to the results of the 5\% licorice gel against two bacteria (Gram positive, S. aureus, and Gram negative, P.aeruginosa, and one yeast (C.albicans) by using disc-diffusion method, all the investigated licorice concentrations inhibited the growth of S.aureus and C.albicans. However, there was no activity against Gramnegative bacteria P.aeruginosa, which showed higher resistance to the prepared gel than the tested Grampositive bacteria and yeast (Table 4). This could be explained by the differences in the cell membrane structure. ${ }^{28}$ Our results indicated that the prepared licorice gel had higher anti-microbial activity against Candida species than against the tested bacterial species with zone of inhibition for $S$. aureus ranged from 0-25 mm, while for C.albicans the zone of inhibition ranged from 12$30 \mathrm{~mm}$. Supportive results were obtained from work conducted by Karahana et al in 2016. ${ }^{29}$ Their work showed that using disc agar diffusion method, licorice has anti-bacterial activity and the zone of inhibition for S.aureus ranged from $12-19 \mathrm{~mm}$, while for P.aeruginosa there was no anti-bacterial activity. Geetha and Anitha in $2013 .{ }^{30}$ showed that the zone of inhibition for C. albicans when treated by licorice ranged from 9-20 $\mathrm{mm}$ in diameter.

\section{CONCLUSION}

It is obvious that licorice gel can be prepared easily from the ethanol/ water licorice extract. Among the several formulas that prepared, two formulas were selected for further investigations. The selected gel 
formula exhibited good physical and chemical characteristics including the appearance, consistency, $\mathrm{pH}$ and skin irritancy test. In addition, it demonstrated good stability at room temperature without noticeable change after storing for four months. The selected formula exhibited good anti-microbial effect against two of the most common causes of skin infections: the gram positive bacteria (S.aureus) and one of the common types of yeast (C.albicans).

\section{ACKNOWLEDGEMENTS}

The authors are grateful to the College of Pharmacy, University of Mosul in Iraq for their support in performing this research. Special thanks to Dr. Hanan Kassab, Assistant professor at College of Pharmacy, University of Baghdad, Baghdad, Iraq for her

\section{REFERENCES}

1. Hesham R. Omar, Irina Komarova, Mohamed El-Ghonemi, et al. Licorice abuse: time to send a warning message. Therapeutic Advances in Endocrinology and Metabolism. 2012; (4).

2. Du JR, Long FY, Chen C. Research progress on natural triterpenoid
To conclude, a topical gel of licorice could be easily prepared and stored at room temperature. It could be considered as a suitable candidate of new herbal antimicrobial pharmaceutical preparation and could be used as an efficient alternative to the conventional anti-microbial topical gel preparations to come onto the market.

unlimited cooperation. The researchers would like to thank Dr. Hadeel M. Al-Hashemi, lecturer at Veterinary Medicine College, University of Mosul for her great efforts and help.

saponins in the chemoprevention and chemotherapy of cancer. In The enzymes 2014 Jan 1 (Vol. 36, pp. 95130). Academic Press

3. Trupti W. Charpe, Virendra K. Rathod. Extraction of glycyrrhizic acid from licorice root using ultrasound: Process intensification studies. Chemical Engineering and Processing. 2012; 54:37- 41. 
4. Al-Ani WN, Al-Haliem SM, licorice (Glycyrrhizaglabra) roots. Tawfik NO. Evaluation of the Separation and Purification Antibacterial Activity of Citrus Technology, 2008; (63):539-545. Juices: An In Vitro Study. AlRafidain Dent J. 2010; 10(2):376-382. 5. Hamdoon AA, Al-khashab EM, Alhashemi HM. Effect of saponin extract of Glycyrrihiza glabra in activity of hepatic enzymes and some biochemical parameters in serum of adults ovariectomized female rats. Iraqi Journal of Veterinary Sciences. 2020 May 13;34(2):411-5.

6. Malvania EA, Sharma AS, Sheth SA, Rathod S, Chovatia NR, Kachwala MS. In Vitro Analysis of Licorice (Glycyrrhiza glabra) Root Extract Activity on Streptococcus mutans in Comparison to Chlorhexidine and Fluoride Mouthwash. The Journal of Contemporary Dental Practice. 2019 Dec 1;20(12):1390.

7. Salehi M, Saeedi M, Ehsani H, Sharifian A, Moosazadeh M, RostamkalaeiS, et al. Analyzing GlycyrrhizaGlabra (Licorice) Extract Efficacy in Recurrent Aphthous Stomatitis Recovery. J Res Med Dent Sci. 2018; 6 (1):68-75.

\section{Mamata Mukhopadhyay,} PalashPanja. A novel process for extraction of natural sweetener from

9. Moghimipour E, Ameri A, Handali S, Ramezani Z, Azemi ME, SadaghiNegad B. In-vitroevaluation of antibacterial activity of glycyrrhizaglabraandacanthopyllums quarrusomtotal saponins. Res J Pharmaceutical BiolChem Sci. 2015; 6(5): 764-768.

10. Shirazi MH, Ranjbar R, Eshraghi S, Sadeghi G, Jonaidi N, Bazzaz N, et al. An evaluation of antibacterial activity of glycyrrhizaglabra extract on the growth of the Samonella, Shigella and ETSC E. coli. J Biol Sci. 2007; 7(5):827-829.

11. MridulHaque, Anil Kumar Singh, Santosh K Maurya, Ankit Seth. Formulation development, physicochemical characterization and evaluation of anti-microbial activity of herbal tooth gel. J Chem Pharm. Res. 2014; 6(3):1279-128.

12. Venus DilshadNajeeb, Ali Sultan Al-Refai. Antibacterial effect and healing potentialof topically applied licorice root extract on experimentally induced oral wounds in rabbits. Saudi Journal of Oral Sciences. 2015; 2 (1): 10-13. 
13. Dheeraj T Baviskar, Yogeshkumar A Biranwar, Kapil R Bare, Venkatesh B Parik, Mangesh K Sapate,Dinesh K Jain. In Vitro and In Vivo Evaluation of Diclofenac Sodium Gel Prepared with Cellulose Ether and Carbopol 934P. Trop J PharmRes.2013;12 (4):489-494.

14. M Saeedi, K Morteza-Semnani, M$\mathrm{R}$ Ghoreishi. The treatment of atopic dermatitis with licorice gel. Journal of Dermatological Treatment. 2003; 14 : $1-3$.

15. Al-NimaA, Al-kotaji M, Al-Iraqi O, Ali Z. Preparation and Evaluation of Ultrasound Transmission Gel. Asian journal of pharmaceutical and clinical research, 2019vol (12), issue 1.

16.Tuğcu-Demiröz F, Acartürk F, AykutÖzkul. Preparation and characterization of bioadhesive controlled-release gels of cidofovir for vaginal delivery. J Biomater Sci Polym Ed. 2015; 26 (17): 1237-55.

17. Tuğcu-Demiröz, F., Acartürk, F., Erdoğan, D. Development of longacting bioadhesive vaginal gels of oxybutynin: formulation, in vitro and in vivo evaluations. Int J Pharm. 2013 Nov 30; 457 (1): 25-39.

18. SachinkumarPatil, AtulKadam, SandipBandgar, ShitalkumarPatil.
Formulation and Evaluation of an In Situ Gel for Ocular Drug Delivery of Anticonjunctival Drug. Cellulose Chem. Technol.2015; 49(1): 35-40.

19. D. Prasanthi, P. K. Lakshmi. Optimisation of Transdermal Gel Formulations of Tolterodine Tartrate by Experimental Design.Turk. J Pharm Sci. 2013; 10 (2): 273-286.

20. Nawaz A, Jan Su, Khan NR, Hussain A, Khan GM. Formulation and in vitro evaluation of clotrimazole gel containing almond oil and tween 80 as penetration enhancer for topical application. Pak J Pharm Sci 2013; 26 : 617-622.

21. Shukr MH, Metwally GF. Evaluation of topical gel bases formulated with various essential oils for antibacterial activity against methicillin-resistant Staphylococcus aureus trop. J Pharm Res 2013;12:87784.

22. Khullar R, Kumar D, Seth N, Saini S. Formulation and evaluation of mefenamic acid emulgel for topical delivery. Saudi Pharm J 2012;20:63-7. 23. Hussain A, Khan GM, Shah SU, Shah KU, Rahim N, Wahab A. Development of a novel ketoprofen transdermal patch: Effect of almond oil as penetration enhancers on in-vitro 
and ex-vivo penetration of ketoprofen through rabbit skin. Pak J Pharm Sci 2012; 25: 227-232.

24. Chakole CM, Shende MA, Khadatkar SN. Formulation and development of novel combined halobetasol propionate and fusidic acid ointment. Int J Chem Tech Res. 2009; 1: 103-16.

25. Santosh Kumar Maurya, Ankit Seth. Formulation development, physico-chemical characterization and evaluation of anti-microbial activity of herbal tooth gel. Journal of Chemical and Pharmaceutical Research, 2014, 6(3):1279-1285.

26. Chaudhary H, Rohilla A, Rathee P, Kumar V. Optimization and formulation design of carbopol loaded piroxicam gel using novel penetration enhancers. Int $\mathrm{J}$ BiolMacromol 2013;55:246-53.

27. Laxmi RJ, Karthikeyan R, Babu PS, Babu RV. Formulation and evaluation of antipsoriatic gel using natural excipients. J Acute Dis.2013;1:115-21.
28. Najeeb VD, Al-Refai AS. Antibacterial effect and healing potential of topically applied licorice root extract on experimentally induced oral wounds in rabbit. S J Oral Sci. 2015; 2(1): 10-13.

29. Karahana F, Avsarb C, Ozyigitc II, Berberb I. Antimicrobial and antioxidant activities of medicinal plant glycyrrhizaglabra var. glandulifera from different habitats. Biotech \&Biotechnological Euipment. 2016; 30(4): 797-804.

30. Geetha RV, Anitha R. In vitro Evaluation of antimycotic activity of ethanolic extract of glycyrrhizaglabra. Asia J Pharmaceutical Clin Res. 2013; 6(3): 205-206.

31. Trupti W. Charpe, Virendra K. Rathod. Extraction of glycyrrhizic acid from licorice root using ultrasound: Process intensification studies. Chemical Engineering and Processing. 2012; 54:37-41. 